\title{
RELAÇÃO ENTRE ESTADO NUTRICIONAL DA GESTANTE, FUMO DURANTE A GRAVIDEZ, CRESCIMENTO FETAL E NO PRIMEIRO ANO DE VIDA
}

\author{
Arnaldo Augusto Franco de Siqueira * \\ Jair Licio Ferreira San tos ** \\ Jane Ferreira da Silva ***
}

SIQUEIRA, A.A.F. de et al. Relação entre estado nutricional da gestante, fumo durante a gravidez, crescimento fetal e no primeiro ano de vida. Rev.Saúde públ., S. Paulo, 20:000-00, 1986.

RESUMO: Estudou-se a relação entre estado nutricional e hábito de fumar maternos, peso do recém-nascido ao nascer e crescimento no primeiro ano de vida num grupo de 1.066 gestantes de baixo nível sócio-econômico. Os filhos de mulheres fumantes apresentaram pesos significativamente menores que os filhos de não-fumantes, quer fossem suas mães, normais ou obesas. Também foi possível verificar que filhos de mulheres desnutridas pesaram significativamente menos que filhos de mulheres normais e estes que os de obesas. O prejuízo no peso dos filhos de mulheres fumantes manteve-se apenas até os 3 meses de idade, ao passo que até os 9 meses os filhos de mulheres desnutridas pesaram significativamente menos que as demais crianças, mostrando que, mesmo em gestantes de baixa renda, o efeito do fumo sobre o crescimento se restringe ao ambiente intra-uterino. Já o efeito da desnutrição materna é mais duradouro nessa população. Verificou-se que houve uma associação negativa entre estado nutricional e hábito de fumar maternos, sugerindo que, ao menos em parte, o efeito do tabagismo matemo sobre o concepto pode ser intermediado pelo estado nutricional.

UNITERMOS: Gravidez. Estado nutriconal. Tabagismo. Peso ao nascer. Peso por idade. Desenvolvimento fetal. Desenvolvimento infantil.

\section{INTRODUÇÃO}

E tido como certo que a desnutrição materna prejudica o crescimento fetal, em peso e altura, contribui para aumentar as taxas de morbidade e mortalidade perinatais e, se houver suplementação alimentar durante a gravidez, estes efeitos são minimizados $8,27,28$

Alguns autores concluíram que fatores nutricionais imediatamente relacionados à gestação ou anteriores a ela têm grande importância na ocorrência de desnutrição intra-uterina 5,26,27.

Outras variáveis tais como: idade gestacional, paridade, idade materna, patologias presentes na gravidez, alterações placentárias, anomalias congênitas, peso pré-gestacional, ganho de peso materno, condições sócio-econômico-culturais, hábito de fumar, alcoolismo e uso de drogas podem influenciar, direta ou indiretamente, o crescimento intra-uterino e pós-natal22.

Os efeitos prejudiciais do fumo sobre a gestação são conhecidos há bastante tempo.

Em 1935, Campbell4 observou que mulheres que fumavam excessivamente tisham bebês prejudicados pelo resultado do envenenamento crônico pela nicotina.
A primeira contribuição de grande valor a esse respeito ocorreu em 1957, quando Simpson39, na Califómia, estudou a distribuição dos pesos de recém-nascidos filhos de mães fumantes e encontrou que estas crianças pesavam, em média, $200 \mathrm{~g}$ a menos do que crianças de mães não-fumantes.

Desde então, vários estudos foram realizados associando o fumo com problemas tais como: diminuição do peso ao nascer, aumento da mortalidade infantil, maiores taxas de aborto espontâneo, prematuridade, morbidade, retardo no crescimento fetal, pré-eclâmpsia, anomalias placentárias e congênitas $3,11,17,18,20,24,46$

Embora diversos autores tenham relatado que mães fumantes são mais suscetiveis de terem gestaçбes de menor duração 18,45 , a menor média de peso ao nascer de bebês de mães fumantes não pode ser explicada pela gestação mais curta.

Alguns estudos mostraram que os efeitos tóxicos do fumo apresentaram um impacto no crescimento fetal a partir do 4 ! mês de gestação ${ }^{1.37}$.

Porém, Rosemberg 33 afirmou que o peso do feto aumenta de maneira semelhante até as útlimas semanas de gestação mas, a partir da $34^{\mathrm{a}}$ semana,

* Do Departamento de Saúde Materno-Infantil da Faculdade de Saúde Pública da Universidade de São Paulo - Av. Dr. Arnaldo, 715 - 01255 - São Paulo, SP - Brasil.

* Do Departamento de Epidemiologia da Faculdade de Saúde Pública da Universidade de São Paulo - Av. Dr. Arnaldo, 715 - 01255 - São Paulo, SP - Brasil.

* Da Real Benemérita Sociedade Portuguesa de Beneficiência - Rua Maestro Cardim, 769 - 01323 - São Paulo, SP - Brasil. 
aproximadamente, começa a ocorrer redução no peso dos fetos de mães fumantes e, quanto mais longa a duração da gestação, maior a diferença ponderal em prejuízo desses fetos.

As investigações de Silverman 38 e de Wainright 44 mostraram que mulheres que continuam fumando têm uma redução no peso ao nascer dos filhos nascidos sucessivamente, enquanto que mulheres que não fumam mostram aumento no mesmo peso.

Vários estudos realizados referem que o retardo no crescimento intra-uterino aumenta com o número de cigarros fumados, independentemente da nutrição materna $15,20,25$, contrariando a sugestão de Garn e col. 12 de que a obesidade materna neutraliza o efeito do fumo sobre o feto.

A prega cutânea é um indicador da gordura subcutânea $e$, certamente, as condições maternas durante a gravidez afetam a prega cutânea do be bê. D'Souza e col. ${ }^{10}$ realizaram um estudo para investigar os efeitos do fumo sobre a prega cutânea, ganho de peso materno e peso do recém-nascido ao nascer. Encontraram que gestantes que fumavam de 40 a 60 cigarros por dia ganhavam significativamente menos peso do que as não-fumantes, sendo que os bebês nascidos de mães fumantes apresentavam peso mais baixo ao nascer e circunferência cefálica menor do que os bebês de não-fumantes, mas a prega cutânea era semelhante. A presença de uma camada normal de gordura subcutânea nos bebês de mães fumantes sugere que o retardo no crescimento fetal não é causado por diferença nutricional.

Recentemente tem surgido interesse com relação aos mecanismos pelos quais o cigarro induz ao retardo no crescimento fetal e os efeitos subseqüentes sobre o crescimento e desenvolvimento de crianças 6 .

Segundo Naeye ${ }^{25}$, há especulações de que o prejuízo no crescimento fetal pode ser devido à: ingestão alimentar inadequada da gestante; concentração de carboxihemoglobina, provocando hipóxia e redução da difusão útero-placentária; diferenças genéticas entre fumantes e não-fumantes; baixo peso pré-gestacional; e alterações de pressão sanguínea de fumantes. Para Rosemberg33 a explicação mais plausível do efeito nocivo do cigarro sobre o feto é o nível de monóxido de carbono no sangue matemo $e$, em conseqüència, na circulação da placenta e no feto, gerando certo grau de hipóxia. Assim, a diminuição de oxigênio e aumento de carboxihemoglobina, decorrente dos altos níveis de monóxido de carbono, prejudica o fe to pela hipóxia, que justifica a redução no crescimento do feto, esclarecendo o menor peso ao nascer.

Outra hipótese é que o cigarro induz à perda do apetite nas mulheres que passam a comer menos, ganhando menos peso, podendo resultar em be bês menores.
Davies e col. 17 mostraram que gestantes fumantes ganham menos peso durante a gestação, sugerindo que o fumo pode reduzir o apetite da mãe e, assim, resultar em uma desnutrição que poderá causar restriçōes ao crescimento do feto.

Entretanto, $\mathrm{Papoz}$ e col. 30 notaram que a ingestão calórica e o ganho de peso foram maiores em mulheres que fumaram durante toda a gravidez e mais baixos nas que não fumaram. $O$ peso ao nascer entre conceptos de fumantes e não-fumantes não apresentou diferenças significativas, apoiando a afirmação de Rush ${ }^{34}$, de que o aumento na ingestão alimentar de fumantes pode inverter ou minimizar os efeitos do fumo sobre o retardo no crescimento.

Diversos outros autores constataram que o retardo no crescimento fetal de filhos de mães fumantes não é devido a menor ingestão de alimentos $10,15,19,43$.

Contrariando muitos autores Yerushalmy 45 afirmava que a relação entre tabagismo e baixo peso ao nascer era devida à fumante e não ao fumo, ou seja, era devida a diferenças constitucionais das fumantes, quando comparadas a não-fumantes. Poucos au tores adotam esta linha, sendo que a maioria acredita em um efeito direto do fumo materno sobre o crescimento intra-u terino.

Seja por qual mecanismo for, o efeito do fumo materno sobre o crescimento fetal é observado através do menor peso ao nascer. Logo, é importante saber se este efeito é passageiro e desaparece logo depois do nascimento, ou se o fumo exerce um efeito a longo prazo sobre o desenvolvimento sub. seqüente na infância.

Russel e col. 35 verificaram que o ganho de peso e o aumento no perímetro cefálico em bebês de fumantes era siginificativamente maior que em be bês de não-fumantes, mas este aumento de velocidade de crescimento cessou por volta de um ano embora os bebês de fumantes ainda fossem menores que os de não-fumantes.

Hardy e Mellits ${ }^{16}$ acreditam que o retardo no crescimento intra-uterino se estende até a vida pós-natal.

Em recente estudo Siqueira e col. 41 verificaram, a partir de dados de um grupo de gestantes e crian. ças de nível sócio-econômico elevado, que recém. - nascidos filhos de mães fumantes eram sempre menores que filhos de mães não-fumantes, quer fossem as mães desnutridas, normais ou obesas. No entanto, o crescimento de filhos de fumantes e de filhos de mulheres desnutridas foi maior que o das demais crianças ao longo do primeiro ano de vida de tal sorte que, ao final desse período, não havia dife renças significativas no peso e altura dessas crianças quando comparadas aos das demais. 
SIQUEJRA, A.A.F. de et al. Relação entre estado nutricional da gestante, fumo durante a gravidez, crescimento fetal e no primeiro ano de vida. Rev. Saúde públ., S. Paulo, 20:421-34, 1986.

Torna-se importante verificar se os resultados obtidos por Siqueira e col. 41 poderão ou não repetir-se em uma população de nivel sócio-econômico mais baixo

Assim, o presente estudo pretendeu verificar a relação entre estado nutricional da gestante, fumo durante a gravidez e crescimento fetal e no primeiro ano de vida, num grupo de gestantes de baixo nível sócio-econômico e seus filhos.

\section{METODOLOGIA}

\section{População}

O presente estudo foi realizado a partir de fichas familiares, contendo dados referentes ao pré-natal de gestantes e do acompanhamento pós-natal de seus filhos durante o primeiro ano de vida. Todas as fichas analisadas referiam-se ao controle de atendimento de 3 postos de saúde, pertencentes à $\mathrm{Re}$ gional Norte do Departamento de Saúde da Comunidade, da Secretaria Municipal de Higiene e Saúde de São Paulo. Nestes 3 postos já havia sido implantado o controle programático das fichas familiares, motivo de sua escolha.

Levantaram-se todas as fichas familiares do período de $1 / 1 / 81$ a $15 / 9 / 83$, num total de 5.000 , das quais apenas 1.066 puderam ser selecionadas para o estudo por satisfazerem os seguintes critérios de inclusão:

- cada criança deveria ter, pelo menos, 3 consultas entre 0 a 12 meses, e nestas deveriam constar peso e data em cada consulta;

- as fichas das gestantes deveriam conter dados sobre peso e estatura materna.

Não houve, na opinião dos autores, vício de seleção, uma vez que não se pretendeu, em nenhum momento, estender os achados da população efetivamente estudada para toda a população, seja de ges. tantes atendidas no serviço, seja das demais gestantes de São Paulo ou do Brasil.

Tratando-se, de um estudo tipo caso-controle, as relações entre fumo, nutrição e crescimento fetal ocorridas em uma gestante e seu concepto dificilmente teriam alguma relação com o que ocorre com outra gestante e seu concepto.

\section{Procedimentos}

Das fichas familiares, obtiveram-se os seguintes dados referentes a:

1. Gestante

- Estatura materna

- Peso na última consulta de pré-natal

- Hábito de fumar

- Idade gestacional.
Em relação ao hábito de fumar, considerou-se fumante a mãe que fumou na gravidez, independente da época e número de cigarros fumados.

2. Criança

- Sexo

- Data de nascimento

- Peso ao nascer

- Data e peso em cada consulta até 12 meses de idade.

De posse dos dados do peso, altura e idade gestacional na última consulta de pré-natal, efetuou-se a avaliação do estado nutricional das gestantes, com o auxílio das curvas de ganho de peso de gestantes, de Siqueira e col. 42 .

Classificaram-se, então, as gestantes em desnutridas, normais e obesas.

Os dados referentes ao peso ao nascer e durante 0 primeiro ano de vida foram distribuídos segundo o estado nutricional e/ou hábito de fumar da mãe.

Observou-se a evolução do peso durante o primeiro ano de vida, segundo as variáveis idade, sexo da criança, estado nutricional e hábito de fumar maternos.

Para apreciar as diferenças entre as médias de peso do recém-nascido e da criança ao longo do primeiro ano de vida utilizou-se a estatística $\mathrm{Z}$ monocaudal com nivel de significância de $5 \%{ }^{9}$.

A associação entre variáveis qualitativas foi analisada estatisticamente pelo método do qui-quadrado $\left(\mathrm{X}^{2}\right)$ ao nivel de significância de $5 \% 9$.

Nos casos em que foi necessário testar a igualdade de mais de duas médias foi utilizada a análise de variância a um critério, modelo fixo, conforme especificação de Dixon e Massey9.

Para aquelas variáveis, nas quais a análise de variância detectou diferenças significativas entre as médias, foram aplicados contrastes de Scheffé36, com o objetivo de individualizar tais diferenças.

Para a associação da adequação do peso do recém-nascido com as demais variáveis adotou-se como adequado o peso igual ou superior a $3.000 \mathrm{~g}$.

\section{RESULTADOS}

No período em estudo, os 1.066 prontuários referiam-se a crianças, e respectivas mães, que satisfizeram os criterios de inclusão descritos na metodologia, sendo 555 do sexo masculino e 511 do sexo feminino.

A Tabela 1 mostrou que o peso médio dos recém- nascidos do sexo masculino foi significativamente maior que o peso médio dos recém-nascidos do 
SIQUEIRA, A.A.F. de et al. Relação entre estado nutricional da gestante, fumo durante a gravidez, crescimento fetal e no primeiro ano de vida. Rev. Saúde públ. S. Paulo, $20: 421-34,1986$.

sexo feminino. Este resultado está de acordo com a maioria dos trabalhos da literatura especializa$\mathrm{da}^{2}, 40,41$.

Ao subdividir os recém-nascidos quanto à adequação do peso ao nascer, conforme critério já utilizado em outra publicaçãot1, verificou-se uma associação significativa ao nível de $5 \%$ com o sexo do recém-nascido. Assim, a Tabela 2 mostrou maior proporção de recém-nascidos de peso adequado entre os de sexo masculino (71\%) do que entre os de sexo feminino (29\%). Resultados semelhantes foram obtidos em outro trabalho (Siqueira e col.41).

TABELA 1

Peso Médio do Recém-Nascido Segundo Sexo.

\begin{tabular}{cccc}
\hline Sexo & No de Casos & Peso Médio do R N (g) & Desvio/Padrão \\
\hline Masculino & 555 & 3232,69 & 493,90 \\
Feminino & 511 & 3105,10 & 531,90 \\
\hline
\end{tabular}

$Z$ observado $=4,09$

$Z$ crítico $=1,66$

TABELA 2

Associação Entre Adequação do Peso ao Nascer e Sexo do Recém-nascido.

\begin{tabular}{|c|c|c|c|}
\hline \multirow{2}{*}{ Adequação do Peso } & \multicolumn{2}{|c|}{ Sexo } & \multirow{2}{*}{ Total } \\
\hline & Feminino & Masculino & \\
\hline \multirow[t]{2}{*}{ Adequado } & 299 & 396 & 695 \\
\hline & $(59 \%)$ & $(71 \%)$ & $(65 \%)$ \\
\hline \multirow[t]{2}{*}{ Inadequado } & 212 & 159 & 371 \\
\hline & $(41 \%)$ & $(29 \%)$ & $(36 \%)$ \\
\hline \multirow{2}{*}{ Total } & 511 & 555 & 1066 \\
\hline & $(100 \%)$ & $(100 \%)$ & $(100 \%)$ \\
\hline
\end{tabular}

$\mathrm{X}^{2}$ observado $=19,32$

$\mathrm{X}^{2}$ crítico $=3,84$

TABELA 3

Associação Entre Adequação do Peso ao Nascer e Hábito de Fumar Materno Durante a Gestação. Postos de Saúde - Regional Norte, DSC.I, São Paulo, 1984.

\begin{tabular}{|c|c|c|c|}
\hline \multirow{2}{*}{ Adequação do Peso } & \multicolumn{2}{|c|}{ Hábito de Fumar } & \multirow{2}{*}{ Total } \\
\hline & Fumante & Não-Fumante & \\
\hline \multirow[t]{2}{*}{ Adequado } & 173 & 493 & 666 \\
\hline & $(56 \%)$ & $(68 \%)$ & $(65 \%)$ \\
\hline \multirow[t]{2}{*}{ Inadequado } & 136 & 227 & 363 \\
\hline & $(44 \%)$ & $(32 \%)$ & $(35 \%)$ \\
\hline \multirow{2}{*}{ Total } & 309 & 720 & 1029 * \\
\hline & $(100 \%)$ & $(100 \%)$ & $(100 \%)$ \\
\hline
\end{tabular}

- 37 casos não tinham dados completos.

$\mathrm{X}^{2}$ observado $=14,75$

$\mathrm{X}^{2}$ crítico $=3,84$ 
A Tabela 3 mostrou que quase a metade dos filhos de mães fumantes nasceram com peso inadequado, ao passo que menos de um terço dos fillhos. de não-fumantes pesaram menos de $3.000 \mathrm{~g}$. Esta associação também se revelou significativa ao nível de $5 \%$.
A Tabela 4 mostrou haver uma associação entre o hábito de fumar e o estado nutricional materno, de tal sorte que houve maior proporção de mulheres desnutridas entre as fumantes do que entre as nãofumantes e maior proporção de mulheres obesas entre as não-fumantes do que entre as fumantes.

\section{TABELA 4}

Associação Entre Estado Nutricional Materno e Hábito de Fumar Durante a Gestação. Postos de Saúde - Regional Norte DSC-I, São Paulo, 1984.

\begin{tabular}{|c|c|c|c|}
\hline \multirow{2}{*}{ Estado Nutricional Materno } & \multicolumn{2}{|c|}{ Hábito de Fumar } & \multirow{2}{*}{ Total } \\
\hline & Fumante & Não-Fumante & \\
\hline Desnutrida & $35 \quad(36 \%)$ & $63(64 \%)$ & 98 \\
\hline & $(12 \%)$ & $(9 \%)$ & $(10 \%)$ \\
\hline Normal & $216 \quad(31 \%)$ & $495 \quad(69 \%)$ & 701 \\
\hline & $(76 \%)$ & $(72 \%)$ & $(73 \%)$ \\
\hline Obesa & $35 \begin{array}{c}(21 \%) \\
(12 \%)\end{array}$ & $\begin{array}{c}131(79 \%) \\
(19 \%)\end{array}$ & $\begin{array}{l}166 \\
(17 \%)\end{array}$ \\
\hline$T \circ t$ a 1 & $\begin{array}{c}286(30 \%) \\
(100 \%)\end{array}$ & $\begin{array}{c}679(70 \%) \\
(100 \%)\end{array}$ & $\begin{array}{r}965 * \\
(100 \%)\end{array}$ \\
\hline
\end{tabular}

* 101 casos não tinham dados completos

$\mathrm{X}^{2}$ observado $=8,03$

$\mathrm{X}^{2}$ crítico $=5,99$

A Tabela 5 mostrou que o peso médio dos recém. - nascidos de mães fumantes foi significativamente menor do que o de não-fumantes. Esta diferença se manteve ainda aos 3 meses de idade; porém, aos 6,9 e 12 meses não houve diferença significativa en. tre os pesos de filhos de fumantes e não-fumantes. Siqueira e col. 41 chegaram a resultados semelhantes em trabalho anterior, realizado com uma população de gestantes com características diversas do presente trabalho.

\section{TABELA 5}

Peso Médio das Crianças por Trimestre Até 12 Meses de Idade, Segundo o Hábito de Fumar Materno.

\begin{tabular}{|c|c|c|c|c|c|}
\hline \multirow{4}{*}{ Idade } & \multicolumn{4}{|c|}{ Hábito de fumar } & \multirow{4}{*}{$\mathbf{Z}$} \\
\hline & \multirow{2}{*}{\multicolumn{2}{|c|}{ Fumante }} & \multirow{2}{*}{\multicolumn{2}{|c|}{ Não-fumante }} & \\
\hline & & & & & \\
\hline & n? de casos & $\begin{array}{c}\text { Peso médio } \\
\text { (g) }\end{array}$ & no de casos & $\begin{array}{c}\text { Peso médio } \\
\text { (g) }\end{array}$ & \\
\hline Ao nascer & 309 & $3.047,55$ & 720 & $3.214,19$ & 5,13 (a) \\
\hline $\operatorname{Aos} 3 \mathrm{~m}$ & 224 & $5.675,94$ & 562 & $5.868,51$ & 2,83 (a) \\
\hline $\operatorname{Aos} 6 \mathrm{~m}$ & 159 & $7.594,15$ & 497 & $7.657,67$ & $0,63(\mathrm{~b})$ \\
\hline $\operatorname{Aos} 9 \mathrm{~m}$ & 148 & $8.615,41$ & 429 & $8.726,78$ & 0,99 (b) \\
\hline $\operatorname{Aos} 12 \mathrm{~m}$ & 65 & $9.358,62$ & 171 & $9.380,64$ & 0,12 (b) \\
\hline
\end{tabular}

$a$ - diferença encontrada significativa ao nível de $5 \%$

b - diferença não significativa

No que se refere à relação entre estado nutricional materno na última consulta e o peso médio do recém-nascido ao nascer, as Tabelas 6,7 e 8 mostra- ram que o peso médio de filhos de mulheres desnutridas foi significativamente menor do que o de filhos de mulheres normais e o destes menor que o 
SIQUEIRA, A.A.F. de et al. Relação entre estado nutricional da gestante, fumo durante a gravidez, crescimento fetal e no primeiro ano de vida Rev. Saúde públ., S. Paulo, $20: 421-34,1986$.

de filhos de obesas, conforme já foi descrito por Guimarães 13 e por Siqueira e col.40, em outras casu ísticas.

Quanto à relação entre estado nutricional materno e o peso médio dos recém-nascidos de mães fuman- tes, as Tabelas 9,10 e 11 mostraram que o peso médio ao nascer de filhos de mulheres fumantes e obesas foi significativamente maior que $o$ de filhos de mulheres fumantes normais e desnutridas; não houve, porém, diferença significativa entre os pesos de filhos de fumantes normais e desnutridas.

TABELA 6

Peso Médio do Recém-Nascido Segundo o Estado Nutricional Matemo na Oltima Consulta.

\begin{tabular}{cccc}
\hline Estado Nutricional Materno & No de Casos & Peso médio (g) & Desvio Padrão (g) \\
\hline Desnutridas & 95 & 2952,74 & 542,40 \\
Normais & 725 & 3152,63 & 507,30 \\
Obesas & 176 & 3376,08 & 495,08 \\
\hline
\end{tabular}

TABELA 7

Análise de Variância do Peso Médio do Recém-Nascido, Segundo o Estado Nutricional Matemo na Última Consulta.

\begin{tabular}{|c|c|c|c|c|c|}
\hline $\begin{array}{l}\text { Fonte de } \\
\text { Variação }\end{array}$ & $\begin{array}{l}\text { Soma dos } \\
\text { Quadrados }\end{array}$ & $\begin{array}{c}\text { Graus de } \\
\text { Liberdade }\end{array}$ & $\begin{array}{c}\text { Quadrado } \\
\text { Médio }\end{array}$ & $\begin{array}{r}F \\
\text { Observado }\end{array}$ & Crítico \\
\hline Entre Grupos & $1,153671610 \quad 10^{7}$ & 2,0000 & 5,768358050 & 22,2992 & 3,000 \\
\hline Dentro de Grupos & $2,568691215 \quad 10^{8}$ & 993,000 & 258679,8807 & & \\
\hline Total & $2,684058376 \quad 10^{8}$ & 995,000 & 269754,6107 & & \\
\hline
\end{tabular}

- Estatística $\mathrm{F}$ observada significativa ao nível de $5 \%$

TABELA 8

Contrastes de Scheffé * para a Variável Peso Médio do Recém-nascido, Segundo o Estado Nutricional Materno na Ultima Consulta, ao Nível de Significância de $5 \%$.

\begin{tabular}{lccl}
\hline \multirow{2}{*}{ Médias Comparadas } & \multicolumn{2}{c}{ Contrastes } & Conclusão \\
\cline { 2 - 3 } & Observados & Críticos & \\
\hline Obesas/Normais & 214,45 & 104,69 & Média obesas maior que média normais \\
Obesas/Desnutridas & 414,34 & 151,61 & Média obesas maior que média desnutridas \\
Normais/Desnutridas & 199,89 & 135,94 & Média normais maior que média desnutridas \\
\hline
\end{tabular}

* Scheffé36 (1953)

\section{TABELA 9}

Peso Médio do Recém-Nascido de Mães Fumantes, Segundo o Estado Nutricional Materno.

\begin{tabular}{lccc}
\hline \multirow{2}{*}{ Estado Nutriconal } & \multicolumn{3}{c}{ Fumantes } \\
\cline { 2 - 4 } & No de casos & Peso médio do RN $(\mathrm{g})$ & DP $(\mathrm{g})$ \\
\hline Desnutridas & 35 & 2942,86 & 405,50 \\
Normais & 216 & 3015,05 & 449,18 \\
Obesas & 35 & 3264,00 & 421,29 \\
\hline
\end{tabular}


SIQUEIRA, A.A.F. de et al. Relação entre estado nutricional da gestante, fumo duran te a gravidez, crescimento fetal e no primeiro ano de vida. Rev. Saúde públ., S. Paulo, 20:421-34, 1986.

\section{TABELA 10}

Análise de Variância do Peso Médio do Recém-nascido de Mães Fumantes, Segundo o Estado Nutricional Materno

\begin{tabular}{|c|c|c|c|c|c|}
\hline $\begin{array}{l}\text { Fonte de } \\
\text { Variação }\end{array}$ & $\begin{array}{c}\text { Soma dos } \\
\text { Quadrados }\end{array}$ & $\begin{array}{c}\text { Graus de } \\
\text { Liberdade }\end{array}$ & $\begin{array}{l}\text { Quadrado } \\
\text { Médio }\end{array}$ & ${ }_{\text {Observado }}{ }^{\mathrm{F}}$ & Crítico \\
\hline Entre grupos & $2,217790580 \quad 10^{6}$ & 2,0000 & $1,10889529010^{6}$ & 5,7054 & 3,0000 \\
\hline Dentro de grupos & $5,50037538310^{7}$ & 283,0000 & 194359,5542 & & \\
\hline$T \circ t a l$ & $5,722154441 \quad 10^{7}$ & 285,0000 & 200777,3488 & & \\
\hline
\end{tabular}

* Estatística $\mathrm{F}$ observada significativa ao nível de $5 \%$.

TABELA 11

Contrastes de Scheffé * para a Variável Peso Médio do Recém-nascido de Mães Fumantes, Segundo Estado Nutricional Materno ao Nível de Significância de $5 \%$.

\begin{tabular}{lccl}
\hline \multirow{2}{*}{ Médias Comparadas } & \multicolumn{2}{c}{ Contrastes } & \multicolumn{1}{c}{ Conclusão } \\
\cline { 2 - 3 } & Observados & Críticos & \\
\hline Obesas/Normais & 248,95 & 196,77 & Média obesas maior que média normais \\
Obesas/Desnutridas & 321,14 & 258,14 & Média obesas maior que média desnutridas \\
Normais/Desnutridas & 72,19 & 196,77 & Médias não diferem \\
\hline
\end{tabular}

* Scheffé36 (1953)

No que se refere à relação entre o estado nutricional e o peso médio dos recém-nascidos de mães não-fumantes, as Tabelas 12, 13 e 14 mostraram que o peso médio de filhos de não-fumantes e desnutridas foi significativamente menor que o de filhos de não-fumantes com estado nutricional adequado, e o destes menor que o de filhos de não-fumantes obesas.

TABELA 12

Peso Médio do Recém-Nascido de Mães Não Fumantes, Segundo o Estado Nutricional Materno.

\begin{tabular}{lccc}
\hline \multirow{2}{*}{ Estado Nutricional } & No de Casos & \multicolumn{2}{c}{ Não-Fumantes } \\
\cline { 3 - 4 } & & Peso Médio do RN (g) & DP (g) \\
\hline Desnutridas & 63 & 2990,79 & 611,61 \\
Normais & 485 & 3203,69 & 523,51 \\
Obesas & 131 & 3413,28 & 511,12 \\
\hline
\end{tabular}

TABELA 13

Análise de Variância do Peso Médio do Recém-nascido de Mães Não-fumantes, Segundo o Estado Nutricional Matemo

\begin{tabular}{|c|c|c|c|c|c|}
\hline $\begin{array}{l}\text { Fonte de } \\
\text { Variação }\end{array}$ & $\begin{array}{l}\text { Soma dos } \\
\text { Quadrados }\end{array}$ & $\begin{array}{c}\text { Graus de } \\
\text { Liberdade }\end{array}$ & $\begin{array}{l}\text { Quadrado } \\
\text { Médio }\end{array}$ & Observado & Crítico \\
\hline Entre grupos & $8,31966510 \quad 10^{6}$ & 2,0000 & $4,159833255 \quad 106$ & $14,8159 *$ & 3,0000 \\
\hline Dentro de grupos & $1,897986925 \quad 10^{8}$ & 676,0000 & 280767,2966 & & \\
\hline Total & $1,98118359010^{8}$ & 678,0000 & 292209,9690 & & \\
\hline
\end{tabular}

* Estatística F observada significativa ao nível de $5 \%$. 
SIQUEIRA, A.A.F. de et al. Relação entre estado nutricional da gestante, fumo durante a gravidez, crescimento fetal e no primeiro ano de vida Rev. Saúde públ., S. Paulo, 20:421-34, 1986.

\section{TABELA 14}

Contrastes de Scheffé * para a Variável Peso Médio do Recém-nascido, Segundo Estado Nutricional Materno e Ausência do Hábito de Fumar, Nível de Significância de $5 \%$.

\begin{tabular}{lccc}
\hline \multirow{2}{*}{ Médias Comparadas } & \multicolumn{2}{c}{ Contrastes } & Conclusōes \\
\cline { 2 - 4 } & Observados & Críticos & Média obesas maior que média normais \\
\hline Obesas/Normais & 209,59 & 127,80 & Média obesas maior que média desnutridas \\
Obesas/Desnutridas & 422,79 & 199,00 & Média normais maior que média desnutridas \\
Normais/ Desnutridas & 213,20 & 173,82 & .
\end{tabular}

- Scheffe 36 (1953)

A Tabela 15 mostrou que não houve diferença significativa entre os pesos médios de filhos de desnutridas fumantes e não-fumantes. Porém, as Tabelas 16 e 17 mostraram que os pesos de filhos de mâes fumantes eram significativamente menores que os de filhos de não-fumantes, tanto para filhos de mulheres de estado nutricional normal quanto para filhos de obesas.

Tabela 15

Peso Médio dos Recém-nascidos, Filhos de Mães Desnutridas,

Segundo o Hábito de Fumar Materno Durante a Gestação.

\begin{tabular}{lccc}
\hline Hábito de Fumar & Nọ de Casos & Peso Médio do R N (g) & Desvio Padrão (g) \\
\hline Fumantes & 35 & 2942,86 & 405,50 \\
Não-fumantes & 63 & 2990,79 & 611,61 \\
\hline
\end{tabular}

$\mathrm{Z}$ observado $=0,69$

Diferença encontrada não significativa

Z crítico

- 1,66

Tabela 16

Peso Médio dos Recém-nascidos, Filhos de Mães com Estado Nutricional Adequado, Segundo o Hábito de Fumar Durante a Gestação.

\begin{tabular}{lccc}
\hline Hábito de Fumar & Nọ de Casos & Peso Médio do R N (g) & Desvio Padrão (g) \\
\hline Fumantes & 216 & 3015,05 & 449,18 \\
Não-fumantes & 485 & 3203,69 & 523,51 \\
\hline
\end{tabular}

$\begin{array}{ll}Z \text { observado } & =4,87 \\ Z \text { crítico } & =1,66\end{array} \quad$ Diferença encontrada significativa ao nível de $5 \%$

TABELA 17

Peso Médio dos Recém-nascidos, Filhos de Obesas, Segundo o Hábito de Fumar Durante a Gestação.

\begin{tabular}{lccc}
\hline Hábito de Fumar & No de Casos & Peso Médio do R N (g) & Desvio Padrão (g) \\
\hline Fumantes & 35 & 3264,00 & 421,29 \\
Nāo-fumantes & 131 & 3413,28 & 511,12 \\
\hline
\end{tabular}

$Z$ observado $=2,096$
$Z$ crítico $=1,660$ Diferença encontrada significativa ao nivel de $5 \%$


SIQUEIRA, A.A.F. de et al. Relação entre estado nutricional da gestante, fumo durante a gravidez, crescimento fetal e no primeiro ano de vida. Rev. Saúde públ., S. Paulo, 20:421-34, 1986.

As Tabelas 18,19 e 20 mostraram que o peso médio das crianças de 3 meses de idade, filhos de mães obesas, foi significativamente maior que 0 de filhos de mães desnutridas. Porém, não houve diferença significativa entre os pesos médios das crianças de mães obesas e normais e entre os de filhos de mães normais e desnutridas.

TABELA 18

Peso Médio das Crianças de 3 Meses de Idade, Segundo o Estado Nutricional Matemo

\begin{tabular}{lccc}
\hline \multicolumn{1}{c}{ Estado Nutricional Materno } & No de Casos & Peso Médio da Criança aos 3 M (g) & DP (g) \\
\hline Desnutrida & 75 & 5566,27 & 772,27 \\
Normal & 537 & 5799,68 & 867,74 \\
Obesa & 142 & 5988,27 & 943,94 \\
\hline
\end{tabular}

\section{TABELA 19}

Análise de Variância do Peso Médio das Crianças de 3 Meses de Idade, Segundo o Estado Nutricional Materno

\begin{tabular}{|c|c|c|c|c|c|}
\hline $\begin{array}{l}\text { Fonte de } \\
\text { Variação }\end{array}$ & $\begin{array}{l}\text { Soma dos } \\
\text { Quadrados }\end{array}$ & $\begin{array}{l}\text { Graus de } \\
\text { Liberdade }\end{array}$ & $\begin{array}{l}\text { Quadrado } \\
\text { Médio }\end{array}$ & Observado $^{\mathbf{F}}$ & Crítico \\
\hline Entre grupos & $9,02265770010^{6}$ & 2,0000 & $4,511328850 \quad 10^{6}$ & 5,9090 & 3,0000 \\
\hline Dentro de grupos & $5,73360353210^{8}$ & 751,0000 & 763462,5209 & & \\
\hline$T \circ t$ a 1 & $5,823830109 \quad 10^{8}$ & 753,0000 & 763462,5209 & & \\
\hline
\end{tabular}

Estatística $\mathrm{F}$ observado significativa ao nível de $5 \%$

TABELA 20

Contrastes de Scheffé * para a Variável Peso Médio das Crianças de 3 Meses de Idade, Segundo o Estado Nutricional Materno

\begin{tabular}{lccl}
\hline \multirow{2}{*}{ Médias Comparadas } & \multicolumn{2}{c}{ Contrastes } & \multicolumn{1}{c}{ Conclusōes } \\
\cline { 2 - 4 } & Observados & Críticos & \\
\hline Obesas/Normais & 188,59 & 201,96 & Médias não diferem \\
Obesas/Desnutridas & 422,01 & 305,51 & Média obesas maior que média desnutridas \\
Normais/Desnutridas & 233,02 & 264,16 & Médias não diferem \\
\hline
\end{tabular}

* Scheffé 36 (1953)

Já aos 6 meses de idade, as Tabelas 21,22 e 23 mostraram que o peso medio de filhos de mulheres obesas foi significativamente maior que $o$ de filhos de normais e desnutridas. Porém, não houve diferenças significativas entre os pesos medios de filhos de normais e de desnutridas.

TABELA 21

Peso Médio das Crianças de 6 Meses de Idade, Segundo o Estado Nutricional Matemo

\begin{tabular}{lccc}
\hline Estado Nutricional Materno & No de Casos & Peso Médio das Crianças aos 6 M (g) & DP (g) \\
\hline Desnutrida & 63 & 7351,59 & 996,42 \\
Normal & 499 & 7622,34 & 1071,23 \\
Obesa & 117 & 7980,85 & 1043,97 \\
\hline
\end{tabular}


SIQUEIRA, A.A.F. de et al. Relaçāo entre estado nutricional da gestante, fumo durante a gravidez, crescimento fetal e no primeiro ano de vida. Rev. Saúde públ., S. Paulo, 20:421-34, 1986.

\section{TABELA 22}

Análise de Variância do Peso Médio das Crianças de 6 Meses de Idade, Segundo o Estado Nutricional Materno

\begin{tabular}{|c|c|c|c|c|c|}
\hline $\begin{array}{c}\text { Fontes de } \\
\text { Variação }\end{array}$ & $\begin{array}{c}\text { Soma dos } \\
\text { Quadrados }\end{array}$ & $\begin{array}{l}\text { Graus de } \\
\text { Liberdade }\end{array}$ & $\begin{array}{l}\text { Quadrado } \\
\text { Médio }\end{array}$ & Observado & Crítico \\
\hline Entre grupos & $1,874420640 \quad 10^{7}$ & 2,0000 & $9,372103200 \quad 10^{6}$ & 8,3422 & 3,0000 \\
\hline Dentro de grupos & $7,594574126 \quad 10^{8}$ & 676,0000 & $1,123457711 \quad 106$ & & \\
\hline$T \circ \mathrm{tal}$ & $7,78201619010^{8}$ & 678,0000 & $1,147789999 \quad 106$ & & \\
\hline
\end{tabular}

Estatística F observada significativa ao nivel de $5 \%$

\section{TABELA 23}

Contrastes de Scheffé para a Variável Peso Médio das Crianças de 6 Meses de Idade, Segundo o Estado Nutricional Materno

\begin{tabular}{lccl}
\hline \multirow{2}{*}{ Médias Comparadas } & \multicolumn{2}{c}{ Contrastes } & \multicolumn{1}{c}{ Conclusões } \\
\cline { 2 - 3 } & Observados & Críticos & Média obesas maior que normais \\
\hline Obesas/Normais & 358,51 & 266,69 & Média obesas maior que média desnutridas \\
Obesas/Desnutridas & 629,26 & 405,72 & Médias não diferem \\
Normais/Desnutridas & 270,75 & 347,14 & \\
\hline
\end{tabular}

As Tabelas 24,25 e 26 mostraram que o peso médio das crianças de 9 meses de idade filhos de mães desnutridas foi significativamente menor que o de filhos de normais e obesas; porém, não houve diferença significativa entre os pesos médios de filhos de obesas e normais.

\section{TABELA 24}

Peso Médio das Crianças de 9 Meses de Idade, Segundo o Estado Nutricional Matemo

\begin{tabular}{lccc}
\hline \multicolumn{1}{c}{ Estado Nutricional Materno } & No de Casos & Peso Médio das Crianças aos 9 M (g) & DP (g) \\
\hline Desnutrida & 56 & 8115,36 & 1177,11 \\
Normal & 396 & 8712,21 & 1087,57 \\
Obesa & 105 & 8915,82 & 1216,02 \\
\hline
\end{tabular}

TABELA 25

Análise de Variância do Peso Médio das Crianças de 9 Meses de Idade Segundo o Estado Nutricional Matemo

\begin{tabular}{|c|c|c|c|c|c|}
\hline $\begin{array}{c}\text { Fontes de } \\
\text { Variação }\end{array}$ & $\begin{array}{l}\text { Soma dos } \\
\text { Quadrados }\end{array}$ & $\begin{array}{l}\text { Graus de } \\
\text { Liberdade }\end{array}$ & $\begin{array}{l}\text { Quadrado } \\
\text { Médio }\end{array}$ & Observado $^{F}$ & Crítico \\
\hline Entre grupos & $2,404154510 \quad 10^{7}$ & 2,0000 & $1,202077255 \quad 10^{7}$ & 9,5518 & 3,0000 \\
\hline Dentro de grupos & $6,97199165210^{8}$ & 554,0000 & $1,258483248 \quad 10^{6}$ & & \\
\hline Total & $7,212407103 \quad 10^{8}$ & 556,0000 & $1,297195522 \quad 10^{6}$ & & \\
\hline
\end{tabular}

Estatística F observada significativa ao nível de $5 \%$ 
SIQUEIRA, A.A.F. de et al. Relação entre estado nutricional da gestante, fumo durante a gravidez, crescimento fe tal e no primeiro ano de vida. Rev. Saúde públ., S. Paulo, 20:421-34, 1986.

TABELA 26

Contraste de Scheffé * para a Variável Peso Médio das Crianças de 9 Meses de Idade, Segundo o Estado Nutricional Materno

\begin{tabular}{lccc}
\hline \multirow{2}{*}{ Médias Comparadas } & \multicolumn{2}{c}{ Contrastes } & \\
\cline { 2 - 3 } & Observados & Críticos & \\
\hline Obesas/Normais & 203,61 & 301,63 & Médias não diferem \\
Obesas/Desnutridas & 800,46 & 454,46 & Média obesas maior que média desnutridas \\
Normais/Desnutridas & 596,85 & 392,31 & Média normais maior que média desnutridas \\
\hline
\end{tabular}

- Scheffé36 (1953)

Para as crianças de 12 meses de idade, como as Tabelas 27 e 28 mostraram, não houve diferença significativa entre os pesos de filhos de desnutridas, normais e obesas.

TABELA 27

Peso Médio das Crianças de 12 Meses de Idade, Segundo o Estado Nutricional Matemo

\begin{tabular}{lccc}
\hline \multicolumn{1}{c}{ Estado Nutricional Materno } & No de Casos & Peso Médio da Criança aos 12 M (g) & DP (g) \\
\hline Desnutrida & 28 & 9039,29 & 1321,79 \\
Normal & 166 & 9425,96 & 881,37 \\
Obesa & 41 & 9623,17 & 1081,74 \\
\hline
\end{tabular}

TABELA 28

Análise de Variância do Peso Médio das Crianças de 12 Meses de Idade, Segundo o Estado Nutricional Materno

\begin{tabular}{|c|c|c|c|c|c|}
\hline $\begin{array}{l}\text { Fonte de } \\
\text { Variação }\end{array}$ & $\begin{array}{l}\text { Soma dos } \\
\text { Quadrados }\end{array}$ & $\begin{array}{c}\text { Graus de } \\
\text { Liberdade }\end{array}$ & $\begin{array}{l}\text { Quadrado } \\
\text { Médio }\end{array}$ & Observado & Crítico \\
\hline Entre grupos & $5,74908990010^{6}$ & 2,0000 & $2,874544950 \quad 10^{6}$ & 3,0000 & 3,0000 \\
\hline Dentro de grupos & $2,22151969310^{8}$ & 232,0000 & 957551,5918 & & \\
\hline Total & $2,279010592 \quad 10^{8}$ & 234,0000 & 973736,1504 & & \\
\hline
\end{tabular}

Estatística $\mathrm{F}$ observada não significativa ao nível de $5 \%$

\section{DISCUSSĀO}

Apesar de algumas opiniões encontradas como a de Yerushalmy 46 , que acredita que o menor peso ao nascer de crianças de mães fumantes seja devido a características constitucionais da fumante e não própriamente ao fumo, o presente estudo concorda com as de diversos outros autores que afirmam que o hábito de fumar prejudica diretamente o crescimento fetal $1,10,25,33,37,39$.

No presente trabalho, realizado com dados de uma amostra populacional de gestantes e crianças de nivel sócio-econômico baixo, verificou-se que o hábito de fumar materno prejudicou o peso ao nascer, tanto de filhos de normais quan to de obesas.

Porem, notou-se que o estado nutricional materno tamberm interferiu no crescimento fetal, apoian. do os resultados de Siqueira 40 e Guimarães ${ }^{13}$, em trabalhos anteriores. Assim, o peso ao nascer de filhos de desnutridas era menor que o de normais e, destes, por sua vez, menor que o de obesas, sendo que em cada grupo de filhos de fumantes eram me. nores que os de não-fumantes. Não houve diferenças significativas entre os pesos de filhos de desnutridas fumantes e não-fumantes. Longe de significar que a desnutrição materna protege contra o efeito do hábito de fumar, o fato pode ser devido a que a desnu. trição materna já tenha provocado tal prejuízo ao tamanho do concepto, que a superposição do efeito do fumo torna-se pouco perceptível.

Assim, provavelmente, a obesidade favorece o crescimento fetal, porém não anula os efeitos preju. diciais do hábito de fumar sobre o crescimento intra-uterino. 
A desnutrição e o hábito de fumar prejudicam o crescimento fetal, sendo que provavelmente haja um sinergismo entre estas duas variáveis, o que talvez explique o fato de os filhos de desnutridas fumantes terem apresentado o maior prejuízo no peso.

No entanto, constatou-se uma associação entre o estado nutricional materno e o hábito de fumar uma vez que houve maior proporção de mulheres desnutridas entre as fumantes do que entre as não. - fumantes e maior proporção de obesas entre as não-fumantes do que entre as fumantes. Tal fato vai ao encontro dos resultados de Gam e col. ${ }^{12}$ que sugerem que parte do efeito prejudicial do fumo seja devido ao estado nutricional materno apesar das opiniōes contrárias de Rantakallio 32 , Naeye 23 e Haworth ${ }^{14}$, que encontraram retardo no crescimento fetal de filhos de fumantes independente do peso pré-gestacional e do ganho de peso, não confirmando a hipótese de que o hábito de fumar interfere no estado nutricional materno através da diminuição da ingestão alimentar.

Não apenas o peso médio ao nascer foi prejudicado como também a adequação do peso ao nascer, onde quase a metade de filhos de mães fumantes nasceram com peso inadequado (menor que $3.000 \mathrm{~g}$ ), concordando com os resultados obtidos por Ounsted e col.29.

Embora não tenham sido estudadas no trabalho em questão a morbidade e a mortalidade infantis, os autores sugerem que, como conseqüência direta da maior proporção dos recém-nascidos de peso inadequado entre filhos de fumantes, os riscos de mortalidade e morbidade estejam aumentados.

No Estudo de Mortalidade Perinatal em Ontá. rio 21 foi encontrado aumento de 22 para $28 \%$ o na taxa de mortalidade perinatal de crianças filhas de mães fumantes.

Porém, Rantakallio ${ }^{31}$, estudando o efeito do fumo matemo duran te a gestação sobre a morbidade e mortalidade de crianças recém-nascidas até os 5 anos de idade, verificou que a mortalidade perinatal não foi maior entre filhos de fumantes, mas a mortalidade pós-neonatal, de 28 dias a 5 anos, foi significativamente maior. Assim como também as crianças filhas de fumantes eram com maior frequiência hospitalizadas e permaneciam no hospital por um tempo mais longo do que as crianças filhas de não-fumantes.

Os resultados obtidos quanto ao crescimento no primeiro ano de vida foram semelhantes aos de trabalhos anteriores 40,41 . A diferença no peso médio de filhos de desnutridas manteve-se por mais tempo e as diferenças nos pesos médios entre filhos de fumantes e não-fumantes logo deixaram de ser observadas.

Não se pode deixar de mencionar o fato de que o pequeno número de casos nas idades mais próximas de um ano pode ter influenciado os resultados da análise de variância; porém, como o número de casos diminuiu em todos os grupos, para a mesma casuística, o efeito do fumo não se manteve para os filhos de mães de nível sócio-econômico baixo, não acon tecendo o mesmo para o estado nu tricional materno.

Assim, tais resultados sugerem que o efeito do estado nutricional materno sobre o crescimento intra-uterino e pós-natal, até 12 meses de idade, seja mais duradouro do que o efeito do hábito de fumar durante a gestação.

Porém, mais estudos em nosso meio são necessários para se averiguar a relação entre hábito de fumar e estado nutricional maternos, crescimento e desenvolvimento das crianças acima de 12 meses de idade.

\section{CONCLUSŌES}

1. Houve associação positiva entre o peso ao nascer e o sexo do recém-nascido, havendo maior proporção de recém-nascidos de peso adequado entre os de sexo masculino e de recém-nascidos de peso inadequado entre os de sexo feminino.

2. Houve maior proporção de recém-nascidos de peso inadequado entre os filhos de mulheres fumantes.

3. Os resultados permitem supor que parte do efeito do hábito de fumar sobre o crescimento fetal seja intermediada pelo estado nutricional materno, uma vez que houve associação entre o hábito de fumar e o estado nutriconal materno.

4. A desnutrição materna interferiu no peso ao nascer tanto de filhos de mães fumantes quanto de não-fumantes.

5. A partir da idade de 6 meses deixou de haver diferença significativa entre os pesos de filhos de fumantes e de não-fumantes.

6. O peso ao nascer de filhos de mulheres fumantes foi menor que o de não-fumantes, tanto no caso de mulheres normais quanto obesas.

7. Os recém-nascidos filhos de mulheres desnutridas tiveram peso ao nascer significativamente menor que os filhos de mulheres normais, e os destas, que os filhos de obesas.

3. Essas diferenças perduraram ao longo do primeiro ano de vida. 
SIQUEIRA, A.A.F. de et al. Relação entre estado nutricional da gestante, fumo durante a gravidez, crescimento fe tal e no primeiro ano de vida. Rev. Saúde públ., S. Paulo, 20:421-34, 1986.

SIQUEIRA, A.A.F. de et al. [Relationship between maternal nutritional status, smoking during pregnancy and growth of fetus and of child during first year of life]. Rev. Súde públ., S.Paulo, 20:421-34, 1986.

ABSTRACT: The relationship between maternal nutritional state, maternal cigarette smok ing habits, weight of infants at birth and their growth in the first year of life, in a group of 1,066 pregnant women (and their children) of low socio-economic status was studied. Babies born to smoking mothers weighed significantly less than those born to non-smoking mothers, whether these were normal or even obese women. It was also observed that children born to undernourished mothers weighed significantly less than those born to normal or obese women. Children born to smoking mothers weighed less than the others up to the $3^{\text {rd }}$ month of life only; children born to undemourished women weighed significantly less than the others up to the $9^{\text {th }}$ month of life. Thus, even among pregnant women of low socio-economic status, the effect of smoking on growth is restricted to the intra-uterine environment. The influence of maternal malnutrition is mantained for a longer period in this sample of the population. It was verified that there was a negative association between maternal nutritional status and maternal smoking habits, suggesting that, at least partially, the influence of maternal smoking on the fetus can be mediated by the nutritional status.

UNITERMS: Pregnancy. Nutritional status. Smoking. Birth weight. Weight age. Fetal development. Child development.

\section{REFERENCIAS BIBLIOGRÁFICAS}

1. ABEL, E.L. Smoking during pregnancy: a review of effects on growth and development of offspring. Hum.Biol., 52:593.625, 1980.

2. ARENO, F.B. Contribuição ao estudo de antropometria do recém-nascido. São Paulo, 1984 [Dissertação de Mestrado - Fauldade de Saúde Pública da USP]

3. BUTLER, N.R. et al. Cigarette smoking in pregnancy: its influence on birth weight and perinatal mortality. Brit.med. J., 2: 127-30, 1972.

4. CAMPBELL, A.M. Excessive cigarette smoking in women and its effect upon their reproductive efficiency. J.Mich.med. Soc., 34 : 136, 1935.

5. CASTILlO, E.D. Consideraciones sobre desnutrición materna y desnutrición in útero. Ginec. Obstet.med., 37: $103-18,1975$.

6. CUSHNER, J.M. Maternal behavior and perinatal risks: alcohol, smoking and drugs. Ann.Ref.publ.Hlth, 2: $201-8,1981$.

7. DAVIES, D.P. et ul. Cigarette smoking in pregnancy associations with maternal weight gain and fetal growth. Lancet, $1: 385-7,1976$.

8. DAVIS, C.H. Weight in pregnancy its value as a routine test. Amer.J.Obstet.Gynec., 6:575, 1923.

9. DIXON, W.J. \& MASSEY, F.J. Introduction to statistical analysis. New York, Mac Graw-Hill, 1957.

10. D'SOUZA, S.W. et al. Smoking in pregnancy: association with skinfold thick ness, maternal weight gain and fetal size at birth. Brit.med.J., $282: 1661-3$, 1981.

11. DUFFUS, G.M. \& MC GILIIVRAY, J. The incidence of pre-eclampsia toxemia in smokers and non-smokers. Lancet, 1 : $994-5,1968$.

12. GARN, S.M. et al. Effect of maternal cigarette smoking on Apgar scores. Amer.J.Dis.Child., 135 : 503-6, 1981 .

13. GUIMARÃES, C.M. Estudo da desnutrição materna e da patologia da gestação sobre o crescimento do concepto e da criança no primeiro ano de vida. São Paulo, 1982. [Tese de Doutorado - Faculdade de Saúde Pública da USP]
14. HAWORTH, J.C. Fetal growth retardation in cigarette smoking mother is not due to decreased maternal food intake. Amer.J.Obstet.Gynec., $137: 719-23$, 1980.

15. HAWORTH, J.C. et al. Relation of matemal cigarette smoking, obesity and energy consumption to infant size. Amer.J.Obstet.Gynec., 138 : 1185-8, 1980.

16. HARDY, J.B. \& MELLITS, E.D. Does maternal smoking during pregnancy have a long term effect on the child? Lancet, 2:1332-6, 1972.

17. LOW, J.A. Maternal smoking in human reproduction. Canad.J.publ.Hlth, $72: 390-3,1981$.

18. LOWE, C.R. Effect of mothers smoking habits on birth weight of their children. Brit.med.J., 2:673-6, 1959.

19. MAV, G. Smoking and the fetus. Lancet, $1: 972,1976$.

20. MEYER, M.B. How does maternal smoking affect birth weight and maternal weight gain? Evidence from the Ontario perinatal mortality study. Amer. J.Obstet.Gynec., 131:888-93, 1975.

21. MEYER, M.B. \& TONASCIA, I.A. Maternal smoking pregnancy complications and perinatal mortality. Amer.J.Obstet.Gynaec., $128: 1494-502,1979$.

22. MILLER, H.C. \& MERRIT, T.A. Fetal growth in hu. mans. Chicago, Year Book Medical Publishers, 1979.

23. NAEYE, R.L. Cigarette smoking and pregnancy weight gain. Lancet, 1: 765-6, 1980.

24. NAEYE, R.L. Effects of maternal cigarette smoking on the fetus and placenta. Brit.J.Obstet.Gynaec., 85 : $732 \cdot 7,1978$.

25. NAEYE, RL, Influence of maternal cigarette smoking during pregnancy on fetal and childhood growth. Obstet.Gynaec., 57: 18-21, 1981.

26. NAEYE, R.L. et al. Effects of maternal nutrition on the human fetus. Pediatrics. $52: 484-503,1973$.

27. NATIONAL ACADEMY OF SCIENCE. Food and Nutrition Board. Committee on Maternal Nutrition. Maternal nutrition and the course of pregnancy. Washington, D.C., Academic Press, 1970. 

primeiro ano de vida Rev. Saúde públ., S. Paulo, 20:421-34, 1986.

28. NUTRITION in pregnancy; tenth Study Group of Royal College of Obstetricians and Gynecologists [Editorial]. Lancet, 1 : 1142-3, 1983.

29. OUNSTED, M. et al Smoking during pregnancy its association with other maternal factors and birth weight. Acto obstet. gynaec.scand., $61: 367-71$, 1982.

30. PAPOZ, L.M.S. et al. Maternal smoking and birth weight in relation to dietary habits. Amer.J. Obstet. Gynec., 142: 870-6, 1982.

31. RANTAKALLIO, P. Relationship of maternal smoking to morbidity and mortality of the child up to the age of five. Acta paediat. scand., $67: 621-31,1978$.

32. RANTAKALLIO, $P$, et al. The relationship between birth weight, smoking during pregnancy and maternal weight gain. Amer.J.Epidem., $113: 590-5$, 1981.

33. ROSEMBERG, J, Tabagismo, sério problema de saúde pública. São Paulo, Almed/Ed.USP, 1981.

34. RUSH, D. Examination of the relationship between birth weight, cigarette smoking during pregnancy and matemal weight gain. J.Obstet.Gynaec.Brit. Comm., 81 : 746-52, 1974.

35. RUSSEL, C.S. et al. Smoking in pregnancy, maternal blood pressure, pregnancy outcome, body weight and growth and other related factors. Brit.J. prev. soc. Med., 22:119-26, 1968.

36. SCHEFFE, H. A method for judging all contrasts in the analysis of variance. Biometrika, $40: 87-104$, 1953.

37. SEXTON, M. et al. A clinical trial of change in maternal smoking and its effects on birth weight. J.Amer. med.Ass., 251: $911-5,1984$.
38. SILVERMAN, D.T. Maternal smok ing and birth weight. Amer.J.Epidem., 105:513-21, 1977.

39. SIMPSON, J.W. A preliminary report on cigarette smo$\mathrm{king}$ and the incidence of prematurity. Amer.J.Obs. tet. Gynoec., $73: 808-15,1957$.

40. SIQUEIRA, A.A.F. de Estudo de um conjunto de cur$v$ as antropométricas no diagnóstico do estado nutricional de gestantes e sua relação com o tamanho do recém-nascido. São Paulo, 1979. [ Tese de Doutorado - Faculdade de Saúde Pública da USP ]

41. SIQUEIRÁ, A.A.F. de et al Estado nutricional e hábito de fumar maternos, crescimento intra-uterino e pós-natal. Rev.Saude públ., S.Paulo, 19:37-50, 1985.

42. SIQUEIRA, A.A.F. de et al. Influência da altura e ganho de peso materno e da idade gestacional sobre o peso do recém-nascido: estudo de 3 grupos de gestantes normais. Rev.Saúde públ., S.Paulo, 9:331-42, 1975.

43. SPIRA, A. \& SERVENT, B. Smoking and the fetus. Lancet, $1: 1416-7,1976$.

44. WAINRIGHT, R.L. Change in observed birth weight associated with change in maternal cigarette smoking. Amer.J.Epidem., 117: 668-75, 1983.

45. YERUSHALMY, J. Mother's cigarette smoking and survival of infant. Amer.J.Obstet.Gynec., 88 . $505-18,1964$.

46. YOUNG, K.R. Effects on birth weight of smoking in pregnancy. J.roy.Army med.Corps, 129: 101-3, 1983.

Recebido para publicaçāo em 05/02/1986.

Reapresentado em 30/09/86

Aprovado para publicaçāo èm 08/10/1986. 